\title{
The Plasmatic Aldosterone and C-Reactive Protein Levels, and the Severity of Covid-19: The Dyhor-19 Study
}

\author{
Orianne Villard 1,2,3, ${ }^{+}$, David Morquin 4, ${ }^{+}$, Nicolas Molinari ${ }^{1,5}$, Isabelle Raingeard ${ }^{2}$, \\ Nicolas Nagot ${ }^{1,5}{ }^{(D)}$, Jean-Paul Cristol ${ }^{1,6}$, Boris Jung ${ }^{1,7,8}$, Camille Roubille ${ }^{1,8}$, \\ Vincent Foulongne ${ }^{9}$, Pierre Fesler ${ }^{1,8}$, Sylvain Lamure ${ }^{1,4} \mathbb{D}$, Patrice Taourel ${ }^{1,10}$, \\ Amadou Konate 11,12, Alexandre Thibault Jacques Maria 1,11,13 (D), Alain Makinson 1,4, \\ Ivan Bertchansky 11,12, Romaric Larcher 1,7,8, Kada Klouche ${ }^{1,7,8}$, Vincent Le Moing 1,4, \\ Eric Renard 1,2,3 (D) and Philippe Guilpain 1,11,13,*
}

1 Montpellier School of Medicine, University of Montpellier, 34000 Montpellier, France; orianne.villard@inserm.fr (O.V.); nicolas.molinari@inserm.fr (N.M.); n-nagot@chu-montpellier.fr (N.N.); jp-cristol@chu-montpellier.fr (J.-P.C.); b-jung@chu-montpellier.fr (B.J.); c-roubille@chu-montpellier.fr (C.R.); p-fesler@chu-montpellier.fr (P.F.); s-lamure@chu-montpellier.fr (S.L.); p-taourel@chu-montpellier.fr (P.T.); a-maria@chu-montpellier.fr (A.T.J.M.); a-makinson@chu-montpellier.fr (A.M.); r-larcher@chu-montpellier.fr (R.L.); k-klouche@chu-montpellier.fr (K.K.); v-le_moing@chu-montpellier.fr (V.L.M.); e-renard@chu-montpellier.fr (E.R.)

2 Department of Endocrinology, Diabetes, Nutrition, and INSERM 1411 Clinical Investigation Centre, Montpellier University Hospital, INSERM, 34000 Montpellier, France; i-raingeard@chu-montpellier.fr

3 Institute of Functional Genomics, CNRS, INSERM, University of Montpellier, 34000 Montpellier, France

4 Department of Infectious and Tropical Diseases, Montpellier University Hospital, 34000 Montpellier, France; d-morquin@chu-montpellier.fr

5 IMAG, CNRS, University of Montpellier, Montpellier University Hospital, 34000 Montpellier, France 6 Laboratory of Biochemistry, Montpellier University Hospital, 34000 Montpellier, France

7 Department of Intensive Care Medicine, Montpellier University Hospital, 34000 Montpellier, France

8 PhyMedExp, Université de Montpellier, INSERM, CNRS, 34000 Montpellier, France

9 Laboratory of Virology, Montpellier University Hospital, 34000 Montpellier, France; v-foulongne@chu-montpellier.fr

10 Department of Radiology, Montpellier University Hospital, 34000 Montpellier, France

11 Department of Internal Medicine-Multi-Organ Diseases, Local Referral Center for Auto-Immune Diseases, Montpellier University Hospital, 34000 Montpellier, France; a-konate@chu-montpellier.fr (A.K.);

i-bertchansky@chu-montpellier.fr (I.B.)

12 Department of Internal Medicine-_DIAGORA Unit', Montpellier University Hospital, 34000 Montpellier, France

13 IRMB, INSERM U1183, Montpellier University Hospital, 34000 Montpellier, France

* Correspondence: p-guilpain@chu-montpellier.fr; Tel.: +33-4-67-33-73-32; Fax: +33-4-67-33-72-91

+ These authors contributed equally to this work.

Received: 29 June 2020; Accepted: 17 July 2020; Published: 21 July 2020

check for updates

\begin{abstract}
Background. The new coronavirus SARS-CoV-2, responsible for the Covid-19 pandemic, uses the angiotensin converting enzyme type 2 (ACE2), a physiological inhibitor of the renin angiotensin aldosterone system (RAAS), as a cellular receptor to infect cells. Since the RAAS can induce and modulate pro-inflammatory responses, it could play a key role in the pathophysiology of Covid-19. Thus, we aimed to determine the levels of plasma renin and aldosterone as indicators of RAAS activation in a series of consecutively admitted patients for Covid-19 in our clinic. Methods. Plasma renin and aldosterone levels were measured, among the miscellaneous investigations needed for Covid-19 management, early after admission in our clinic. Disease severity was assessed using a seven-category ordinal scale. Primary outcome of interest was the severity of patients' clinical courses. Results. Forty-four patients were included. At inclusion, 12 patients had mild clinical
\end{abstract}


status, 25 moderate clinical status and 7 severe clinical status. In univariate analyses, aldosterone and C-reactive protein (CRP) levels at inclusion were significantly higher in patients with severe clinical course as compared to those with mild or moderate course ( $p<0.01$ and $p=0.03$, respectively). In multivariate analyses, only aldosterone and CRP levels remained positively associated with severity. We also observed a positive significant correlation between aldosterone and CRP levels among patients with an aldosterone level greater than $102.5 \mathrm{pmol} / \mathrm{L}$. Conclusions. Both plasmatic aldosterone and CRP levels at inclusion are associated with the clinical course of Covid-19. Our findings may open new perspectives in the understanding of the possible role of RAAS for Covid-19 outcome.

Keywords: Covid-19; renin angiotensin aldosterone system; inflammation; endocrine; severity

\section{Introduction}

A new coronavirus called SARS-CoV-2 is responsible for the pandemic of Covid-19, which has led to tens of thousands of deaths around the world so far [1]. Briefly, the disease develops in two phases: the first one is linked to the viral invasion, and the second one consists of a severe acute inflammatory immune response, including a "cytokine storm", which results in severe morbidity and mortality, mainly related to lung injury [2]. In this context, the intensity of the inflammatory process contributes to the disease severity and the plasmatic level of C-reactive protein (CRP) (a biomarker of systemic inflammation) could represent a marker of poor outcomes in Covid-19 patients [3-6].

As observed with the SARS-CoV responsible for SARS 2003 [7], SARS-CoV-2 uses the angiotensin converting enzyme type 2 (ACE2) as a cellular receptor to infect cells. ACE2 is a physiological inhibitor of the renin angiotensin aldosterone system (RAAS) through the catabolism of angiotensin type 2 (Ang2) into angiotensin (1-7) peptide [8]. Ang2 can induce pro-inflammatory responses through its receptor AT1R, while ACE2 reduces anti-inflammatory reactions through its receptor MasR. The assessment of RAAS involvement in the course of Covid-19 in humans is not easy, due to the poor value of Ang2 and angiotensin (1-7) peptide assays in peripheral blood to investigate RAAS and ACE2 in affected patients. Therefore, we explored the levels of plasma renin and aldosterone as indicators of RAAS activation in a series of consecutively admitted patients for Covid-19 in our clinic. Relationships with the severity of disease course were investigated to assess whether RAAS activation could be considered as a biomarker of Covid-19 outcomes.

\section{Patients and Methods}

\subsection{Study Design}

In a series of consecutive patients with Covid-19 diagnosis, hormonal assays including plasma renin and aldosterone levels were performed among the miscellaneous investigations needed for the management of Covid-19, early after admission in our clinic. This study is called Dyhor-19 (dysfunctional hormone regulation during Covid-19) and its protocol was reviewed and approved by the University Hospital of Montpellier Institutional Review Board (IRB-MTP_2020_04_202000441, ClinicalTrials.gov identifier: application in process).

\subsection{Outcome Measures}

The disease severity was assessed using a seven-category ordinal scale (OS) [9], as follows: 1—not hospitalized, no limitation on activities; 2-not hospitalized, limitations on activities; 3-hospitalized, not requiring supplemental oxygen; 4-hospitalized, requiring supplemental oxygen; 5-hospitalized, on non-invasive ventilation or high flow oxygen devices; 6 -hospitalized, on invasive mechanical ventilation or extracorporeal membrane oxygenation (ECMO) and 7-death. 
The primary outcome of interest was the severity of patients' clinical courses during hospitalization, defined as severe for an ordinal scale higher than 4 and corresponding to the transfer to the intensive care unit and death from all-causes. Clinical status using the seven-category ordinal scale was assessed at three different time points: (i) early after admission corresponding to the day of the biological test (Day 0), (ii) two days later (Day 2) and (iii) considering the maximum ordinal scale during the overall period of hospitalization (OS max).

\subsection{Data Collection}

The past medical history, clinical manifestations, comorbidities, treatment strategies, radiologic assessments and laboratory testing on admission were extracted from the electronic medical records. Disease severity was assessed by the OS, as indicated above. Diagnosis of Covid-19 was considered as suspected in patients with typical lung CT-scan lesions and negative SARS-CoV-2 PCR.

Laboratory variables were tested with conventional methods, including routine blood tests: blood count, renal function, inflammatory markers. Determination of CRP was performed using a Cobas8000/e502 ${ }^{\circledR}$ analyzer (Roche Diagnostic, Meylan, France) using the immunoturbidimetric method with reagents from Roche (total CV imprecision results in the laboratory $=3 \%$ ). Renin and aldosterone were determined on an IDS-iSYS multi-discipline automated system (ImmunoDiagnosticSystem, Boldon, United Kingdom) using kits from IDS (total CV imprecision results in the laboratory 5\% and $6 \%$ for renin and aldosterone respectively). A cutoff of $102.5 \mathrm{pmol} / \mathrm{L}$ was the lower limit of plasma aldosterone detection in our condition. Plasma cortisol and adrenocorticotropic hormone (ACTH) levels were measured by automated electrochemiluminescence assays (Cobas 8000, Roche, Basel, Switzerland). Laboratory confirmation of SARS-CoV-2 infection was determined by reverse transcription-PCR from nasopharyngeal swab specimens.

\subsection{Statistical Analyses}

Categorical variables were described as frequency rates and percentages, analyzed using the Chi-squared test or Fisher's exact test. Continuous variables were described using mean and standard deviation (SD). Means for continuous variables were compared using Student t-test or Mann-Whitney test according to the data distribution. Due to skewed distribution, biological variables were presented with median (min-max), and median difference (Hodges-Lehamann estimator). A logistic regression was used for the analysis of the main criteria with odds ratio of disease severity adjusted on the delay from admission. Covariates were selected in a backward selection procedure if $p<0.15$ in the univariate analysis and then presented as adjusted odds ratios (ORs). Potential confounding factors were investigated by testing differences between groups. Studying the relationship between variables was done using Spearman correlation. Statistical analyses were performed using SAS Enterprise Guide, version 7.3 (SAS Institute, Cary, NC, USA) and GraphPad Prism, version 8.4.2 for Mac Os (GraphPad Software, San Diego, CA, USA).

\section{Results}

\subsection{Patients and Clinical Course}

Forty-four patients were included in the study during the period from 26 March to 20 April 2020. Clinical characteristics of the patients are described in Table 1 and Supplementary Materials Table S1. The median age of patients was 66.5 years (interquartile range (IQR, 53 to 75.3 years), and $38.6 \%$ of patients were women. The median interval time between symptom onset and baseline laboratory tests was 8 days (IQR, 5 to 10 days). At inclusion, 12 patients had mild clinical status (OS $\leq 3), 25$ moderate clinical status $(O S=4)$ and 7 severe clinical status $(O S \geq 5)$. On Day 2, 17 patients met a mild clinical status including 2 patients who returned home whereas severe clinical status concerned 12 patients including 2 deaths. During the overall period of hospitalization, estimation of OS max showed that 15 patients (34.1\%) met a severe clinical course including 6 deaths, and 6 patients requiring invasive 
mechanical ventilation. Of the 29 patients with mild or moderate clinical status, 19 required oxygen support. Clinical courses according to the ordinal scale are described in Table 2.

Table 1. Clinical characteristics of patients with Covid-19 according to disease severity classified in two groups: mild/moderate (OS $\max \leq 4)$ and severe (OS $\max \geq 5$ ).

\begin{tabular}{|c|c|c|c|c|}
\hline \multirow[b]{2}{*}{ Patient Characteristics } & \multirow{2}{*}{$\begin{array}{l}\text { Total } \\
(n=44)\end{array}$} & \multicolumn{2}{|c|}{ Disease Severity } & \multirow[b]{2}{*}{$p$-Value } \\
\hline & & $\begin{array}{l}\text { Mild/Moderate } \\
\quad(n=29)\end{array}$ & $\begin{array}{c}\text { Severe } \\
(n=15)\end{array}$ & \\
\hline \multicolumn{5}{|l|}{ Demographics } \\
\hline Age, median (IQR)-years & $66.5(53-75.3)$ & $65(55-75)$ & $70(50.5-75.5)$ & $0.67^{1}$ \\
\hline Female sex-n $(\%)$ & $17(38.6)$ & $9(31)$ & $8(53)$ & $0.15^{2}$ \\
\hline \multicolumn{5}{|l|}{ Diagnosis of Covid-19- $n(\%)$} \\
\hline Positive (SARS-CoV-2 PCR) & $41(93.2)$ & $27(93)$ & $14(93)$ & $1^{3}$ \\
\hline Suspected & $3(6.8)$ & $3(7)$ & $1(7)$ & $1^{3}$ \\
\hline \multicolumn{5}{|l|}{ Signs and symptoms of Covid-19-n (\%) } \\
\hline Fever & $38(86.4)$ & $27(93.1)$ & $11(73.3)$ & $0.16^{3}$ \\
\hline Myalgia & $14(32.6)$ & $9(31)$ & $5(35.7)$ & $1^{3}$ \\
\hline Cough & $28(65.1)$ & $20(69)$ & $8(53.3)$ & $0.31^{2}$ \\
\hline Breathlessness & $24(54.5)$ & $15(51.7)$ & $9(60)$ & $0.60^{2}$ \\
\hline Diarrhea & $12(27.3)$ & $7(24.1)$ & $5(33.3)$ & $0.72^{3}$ \\
\hline Headache & $2(4.5)$ & $1(3.4)$ & $1(6.7)$ & $1^{3}$ \\
\hline Anosmia & $8(18.2)$ & $7(24.1)$ & $1(6.7)$ & $0.23^{3}$ \\
\hline Dysgeusia & $9(20.5)$ & $7(24.1)$ & $2(13.3)$ & $0.7^{3}$ \\
\hline Delay from onset symptoms median (IQR)—day & $8(5-10)$ & $9(6-11)$ & $7(4-8)$ & $0.07^{1}$ \\
\hline \multicolumn{5}{|l|}{ Coexisting conditions $-n(\%)$} \\
\hline Hypertension & $25(56.8)$ & $15(51.7)$ & $10(66.7)$ & $0.34^{2}$ \\
\hline Diabetes mellitus & $15(34.1)$ & $8(27.6)$ & $7(46.7)$ & $0.21^{2}$ \\
\hline Obesity & $11(25)$ & $6(20.7)$ & $5(33.3)$ & $0.47^{3}$ \\
\hline Cardiovascular disease & $16(36.4)$ & $10(34.5)$ & $6(40)$ & $0.72^{2}$ \\
\hline Stroke & $5(11.4)$ & $2(6.9)$ & $3(20)$ & $0.32^{3}$ \\
\hline Chronic kidney disease (eGFR $<60 \mathrm{~mL} / \mathrm{min}$ ) & $7(15.9)$ & $2(6.9)$ & $5(33.3)$ & $0.04^{3}$ \\
\hline Cancer & $8(18.2)$ & 7 (24.1) & $1(6.7)$ & $0.41^{3}$ \\
\hline Dysthyroid disease & $5(11.4)$ & $1(3.4)$ & $4(26.7)$ & $0.04^{3}$ \\
\hline History of organ transplantation & $4(9.1)$ & $1(3.4)$ & $3(20)$ & $0.11^{3}$ \\
\hline Documented acute bacterial infection & $10(22.7)$ & $3(10.3)$ & $7(46.7)$ & $0.02^{3}$ \\
\hline \multicolumn{5}{|l|}{ Long-term anti-hypertensive treatment $-n(\%)$} \\
\hline Angiotensin-converting enzyme inhibitors & $10(22.7)$ & $6(20.7)$ & $4(26.7)$ & $0.71^{2}$ \\
\hline Angiotensin-receptor blockers & $6(13.6)$ & $5(17.2)$ & $1(6.7)$ & $0.65^{2}$ \\
\hline Calcium-channel blockers & $6(13.6)$ & $5(17.2)$ & $1(6.7)$ & $0.65^{2}$ \\
\hline Beta-blockers & $14(31.8)$ & $6(20.7)$ & $8(53.3)$ & $0.04^{2}$ \\
\hline Thiazide diuretics & $3(6.8)$ & $2(6.9)$ & $1(6.7)$ & $1^{2}$ \\
\hline Loop diuretics & $7(15.9)$ & $2(6.9)$ & $5(33.3)$ & $0.04^{2}$ \\
\hline \multicolumn{5}{|l|}{ Care during hospitalization } \\
\hline Duration mean (SD)—day & $13.1(10.9)$ & $10.1(7.5)$ & $18.8(14.1)$ & $0.03^{4}$ \\
\hline Corticosteroid therapy mean (SD)—day & $1.8(3.1)$ & $1.1(2.4)$ & $3.33(3.8)$ & $0.02^{4}$ \\
\hline Use of antibiotic agents-n (\%) & $30(68.2)$ & $16(55.2)$ & $14(93.3)$ & $0.02^{3}$ \\
\hline Use of vasoactive drug-n $(\%)$ & $7(15.91)$ & $0(0.00)$ & $7(46.67)$ & $<0.01^{3}$ \\
\hline
\end{tabular}

OS denotes ordinal scale, IQR interquartile range, $n$ number, eGFR estimated glomerular filtration rate. ${ }^{*}$ Comparison mild/moderate versus severe using Student $t$-test $\left({ }^{1}\right)$. Chi-squared test $\left({ }^{2}\right)$, Fisher's exact test $\left({ }^{3}\right)$ or Mann-Whitney test $\left({ }^{4}\right)$. 
Table 2. Clinical course during hospitalization according to the Covid-19 ordinal scale (mild, moderate, severe gradation).

\begin{tabular}{|c|c|c|c|c|}
\hline \multicolumn{2}{|r|}{ Seven-Category Ordinal Scale } & $\begin{array}{c}\text { Day 0 } \\
\text { (at Inclusion) }\end{array}$ & Day 2 & OS Max \\
\hline \multicolumn{2}{|r|}{ Mild clinical status $-n(\%)$} & $12(27.3)$ & $17(38.6)$ & $10(22.7)$ \\
\hline 1 & Not hospitalized, no limitations on activities- $-n$ & - & - & - \\
\hline 2 & Not hospitalized, limitations on activities- $n$ & - & 2 & - \\
\hline \multirow[t]{2}{*}{3} & Hospitalized, not requiring supplemental oxygen $-n$ & 12 & 15 & 10 \\
\hline & Moderate clinical status $-n(\%)$ & $25(56.8)$ & $15(34.1)$ & $19(43.2)$ \\
\hline \multirow[t]{2}{*}{4} & Hospitalized, requiring supplemental oxygen $-n$ & 25 & 15 & 19 \\
\hline & Severe clinical status- $n(\%)$ & $7(15.9)$ & $12(27.3)$ & $15(34.1)$ \\
\hline 5 & Hospitalized, on non-invasive ventilation or high flow oxygen devices- $n$ & 1 & 5 & 3 \\
\hline 6 & Hospitalized, on invasive mechanical ventilation or $\mathrm{ECMO}-n$ & 6 & 5 & 6 \\
\hline 7 & Death-n & & & \\
\hline
\end{tabular}

At inclusion, on Day 0, patients with severe clinical course (OS max $\geq 5$ ) had more frequently a thyroid or chronic kidney disease, and a concomitant acute bacterial disease, compared to patients with mild or moderate course (OS max $\leq 4)$. A history of hypertension was present in 25 patients (56.8\%). Among antihypertensive treatment, the use of RAAS blockers (angiotensin-converting enzyme inhibitors or angiotensin-receptor blockers) was not different between mild or moderate and severe clinical course and concerned $11(38 \%)$ and 5 patients (33\%) respectively. Beta-blockers and loop diuretics were more frequently used in patients with severe clinical progression (OS max $\geq 5$ ) than in those with mild to moderate clinical course (OS max $\leq 4)$. The most common symptoms on admission were fever $(86.4 \%)$ and cough $(65.1 \%)$ with no differences found between patients with mild or moderate and those with severe clinical course. As expected, treatments during hospitalization including corticosteroid therapy, antibiotics and vasoactive drugs, which were significantly more frequently delivered in patients with severe compared to those with mild or moderate clinical courses.

\subsection{Factors Associated with Disease Severity}

Laboratory findings according to disease severity during the overall period of hospitalization are described in Table 3 between mild/moderate (OS max $\leq 4)$ versus severe (OS max $\geq 5$ ) groups. In univariate analyses, aldosterone levels at inclusion were significantly higher in patients with severe clinical course (OS $\geq 5$ ) (median (min-max), 304.7 (102.5-1360.1) pmol/L) as compared to those with mild or moderate course (OS $\leq 4)(102.5(102.5-540.2) \mathrm{pmol} / \mathrm{L})(p<0.01)$. Of note, at inclusion, potassium levels and aldosterone/renin ratios were not different between these two groups (Figure 1A,C), but in some cases, we observed a trend toward an association between higher levels of aldosterone and lower renin and potassium levels (Figure 1B,D). Concerning cortisol and ACTH levels, no difference was observed between groups. Among common hematological and inflammatory markers at baseline (including lymphocyte, monocyte and eosinophil counts, fibrinogen and D-dimers), CRP at inclusion was significantly higher in patients with severe clinical course (152 (34-389) $\mathrm{mg} / \mathrm{L}$ ) compared to those with mild or moderate course (83 (3-298) $\mathrm{mg} / \mathrm{L}, p=0.03)$. In multivariate analyses including coexisting conditions, long-term anti-hypertensive treatments, care during hospitalization and laboratory findings at inclusion, only aldosterone $(\mathrm{OR}=1.07(1.01-1.14), p=0.033)$ and CRP $(\mathrm{OR}=1.11(1.01-1.22)$, $p=0.024)$ remained positively associated with the severity of clinical course. 
Table 3. Biological findings at inclusion (on Day 0) according to disease severity of patients with Covid-19 infection classified in two groups: mild/moderate (OS max $\leq 4)$ and severe (OS $\max \geq 5)$.

\begin{tabular}{|c|c|c|c|c|c|c|}
\hline \multirow[b]{2}{*}{ Measurements } & \multirow{2}{*}{$\begin{array}{c}\text { Number Data } \\
\text { Available }\end{array}$} & \multirow[b]{2}{*}{ Total } & \multicolumn{2}{|c|}{ Disease Severity } & \multirow{2}{*}{$\begin{array}{l}\text { Median Difference } \\
(95 \% \mathrm{CI})\end{array}$} & \multirow[b]{2}{*}{$p$-Value * } \\
\hline & & & $\begin{array}{l}\text { Mild/Moderate } \\
\quad(n=29)\end{array}$ & $\begin{array}{l}\text { Severe } \\
(n=15)\end{array}$ & & \\
\hline \multicolumn{7}{|l|}{ Univariate analysis } \\
\hline C-reactive protein median (min-max) - $\mathrm{mg} / \mathrm{L}$ & 42 & $92(3-389)$ & $83(3-298)$ & $152(34-389)$ & $65(6 ; 127)$ & $0.03^{1}$ \\
\hline Lymphocyte count median (min-max) $-10^{9} / \mathrm{L}$ & 42 & $1.2(0.2-2.6)$ & $1.1(0.2-2.6)$ & $1.3(0.2-2)$ & $0.2(-0.2 ; 0.5)$ & $0.22^{1}$ \\
\hline Monocyte count median (min-max)-1099/L & 42 & $0.5(0.1-2.3)$ & $0.5(0.1-2.3)$ & $0.5(0.2-1.5)$ & $0.1(-0.1 ; 0.3)$ & $0.42^{1}$ \\
\hline Eosinophil count median (min-max) $-10^{9} / \mathrm{L}$ & 42 & $0.1(0-0.3)$ & $0.1(0-0.3)$ & $0(0-0.3)$ & $-0.02(-0.1 ; 0)$ & $0.11^{1}$ \\
\hline Fibrinogen median (min-max)-g/L & 39 & $5.7(1.2-9.4)$ & $5.6(1.2-9.4)$ & $5.9(3.4-7.6)$ & $0.4(-0.8 ; 1.5)$ & $0.56^{2}$ \\
\hline NT pro-BNP median (min-max)—ng/L & 31 & $423(17-63245)$ & $228(17-16749)$ & $1135(58-63245)$ & $711.50(-17 ; 2065)$ & $0.06^{1}$ \\
\hline Troponin median (min-max)—ng/L & 39 & $13.8(1.5-1596)$ & $12.4(1.5-1596)$ & $23.8(1.5-24)$ & $7.55(-3 ; 68.5)$ & $0.18^{1}$ \\
\hline Ferritin median (min-max) - $\mu \mathrm{g} / \mathrm{L}$ & 35 & $814(133-12460)$ & $603(148-12460)$ & $966(133-7750)$ & $217(-330 ; 965)$ & $0.43^{1}$ \\
\hline Potassium level median (min-max)-mmol/L & 43 & $3.8(2.8-4.8)$ & $3.8(2.8-4.3)$ & $3.8(2.8-4.8)$ & $3.8(2.8 ; 4.8)$ & $0.99^{2}$ \\
\hline Creatinine median (min-max) $-\mu \mathrm{mol} / \mathrm{L}$ & 44 & $74.5(34-949)$ & $71(34-949)$ & $100(40-524)$ & $28(5 ; 54)$ & $0.02^{1}$ \\
\hline eGFR median $(\min -\max )-\mathrm{mL} / \mathrm{min} / 1.73 \mathrm{~m}^{2}$ & 44 & $87.5(3-120)$ & $91(3-120)$ & $59(9-120)$ & $-26(-50 ; 0)$ & $0.05^{1}$ \\
\hline Aldosterone median (min-max) - pmol/L & 44 & $108(102.5-1360.1)$ & $102.5(102.5-540.2)$ & $304.7(102.5-1360.1)$ & $174.5(0 ; 274.2)$ & $<0.01^{1}$ \\
\hline Renin median (min-max) - $\mu$ ui/mL & 44 & $25.8(4.5-1594)$ & $19.00(4.5-495.4)$ & $62.4(9.4-1594)$ & $32.4(9.2 ; 116.9)$ & $<0.01^{1}$ \\
\hline Cortisol median (min-max) $-\mathrm{nmol} / \mathrm{mL}$ & 34 & $471.8(110.9-1320.8)$ & $495.2(110.9-933.2)$ & $377.5(115.6-1320.8)$ & $-91.8(-259.4 ; 90.5)$ & $0.17^{1}$ \\
\hline ACTH median (min-max)- pmol/L & 36 & $3.3(0.2-13.8)$ & $5.5(0.2-13.8)$ & $1.7(0.2-11.1)$ & $-2.9(-5.2 ;-0.3)$ & $0.6^{1}$ \\
\hline
\end{tabular}

eGFR denotes estimated glomerular filtration rate using CKD-EPI equation. * Comparison Mild/Moderate versus Severe using Mann-Whitney test $\left({ }^{1}\right)$ or Student $t$-test $\left({ }^{2}\right)$. 
A.

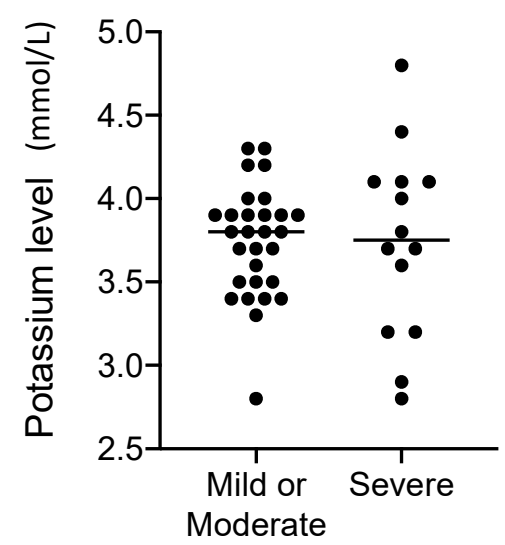

C.

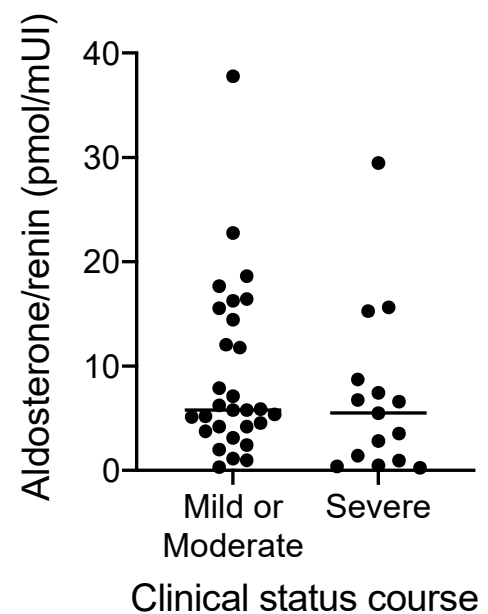

B.

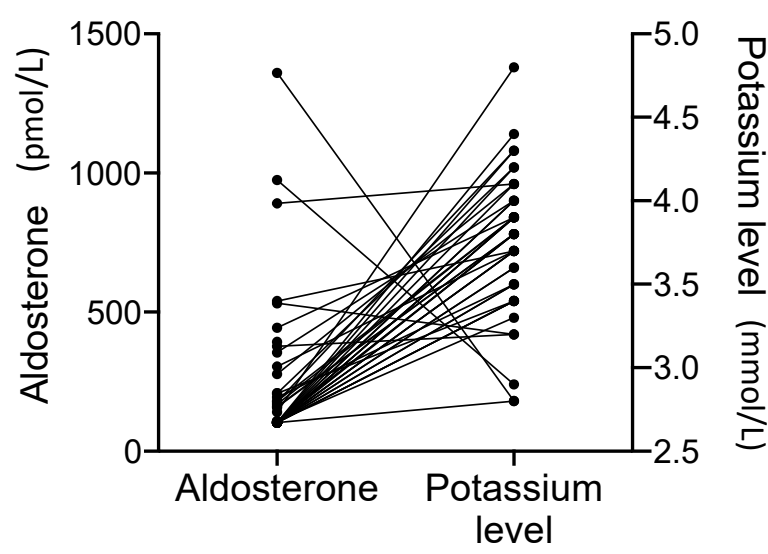

D.

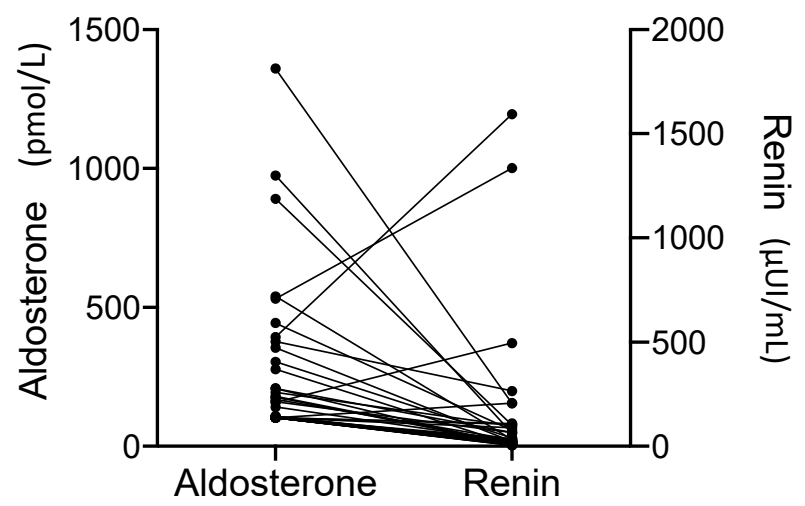

Figure 1. Laboratory findings of RAAS explorations. (A) Plasmatic aldosterone levels according to the maximal ordinal scale (OS) during the overall period of hospitalization classified in two groups: mild or moderate (OS $\max \leq 4$ ) and severe (OS $\max \geq 5$ ).; (B) Plasmatic aldosterone and potassium levels for each patient; (C) Plasmatic aldosterone / renin ratio according to the maximal ordinal scale (OS) during the overall period of hospitalization classified in two groups: mild or moderate (OS max $\leq 4)$ and severe (OS $\max \geq 5$ ).; (D) Aldosterone and renin levels for each patient.

In addition, the plasma aldosterone and CRP levels were examined according to the clinical status at three different time points (Figures 2 and 3A): (i) at inclusion (Day 0), (ii) two days later (Day 2) and (iii) at the maximum ordinal scale during the overall period of hospitalization (OS max). At inclusion, aldosterone levels were not clearly associated with a specific clinical status $(p=0.61)$ (Figure 2A). However, higher aldosterone levels at inclusion were observed in patients with OS at Day 2 or OS $\max \geq 5$ ( $p=0.006$ and $p=0.0013$ respectively) (Figure 2A). Moreover, aldosterone levels were also gradually and significantly increased when we compared clinical status of patients in the three following categories: mild (OS $\leq 3)$, moderate $(O S=4)$ and severe $(O S \geq 5)$ on Day 2 and at $O S$ $\max$ (analysis of variance, $p=0.001$ and $p=0.006$, respectively) (Figure 2B). Notably, similar findings were observed when patients receiving a RAAS blocker were excluded from the analysis on Day 2 and at OS max (analysis of variance, $p=0.008$ and $p=0.025$, respectively) (Supplemental Figure S1). Similarly, these findings were also observed when patients receiving beta-blockers were excluded from the analysis on Day 2 and at OS max (analysis of variance, $p=0.002$ and $p=0.01$, respectively). Notably, we also investigated the effects of age and sex on our findings and did not observe any significant differences between groups. 

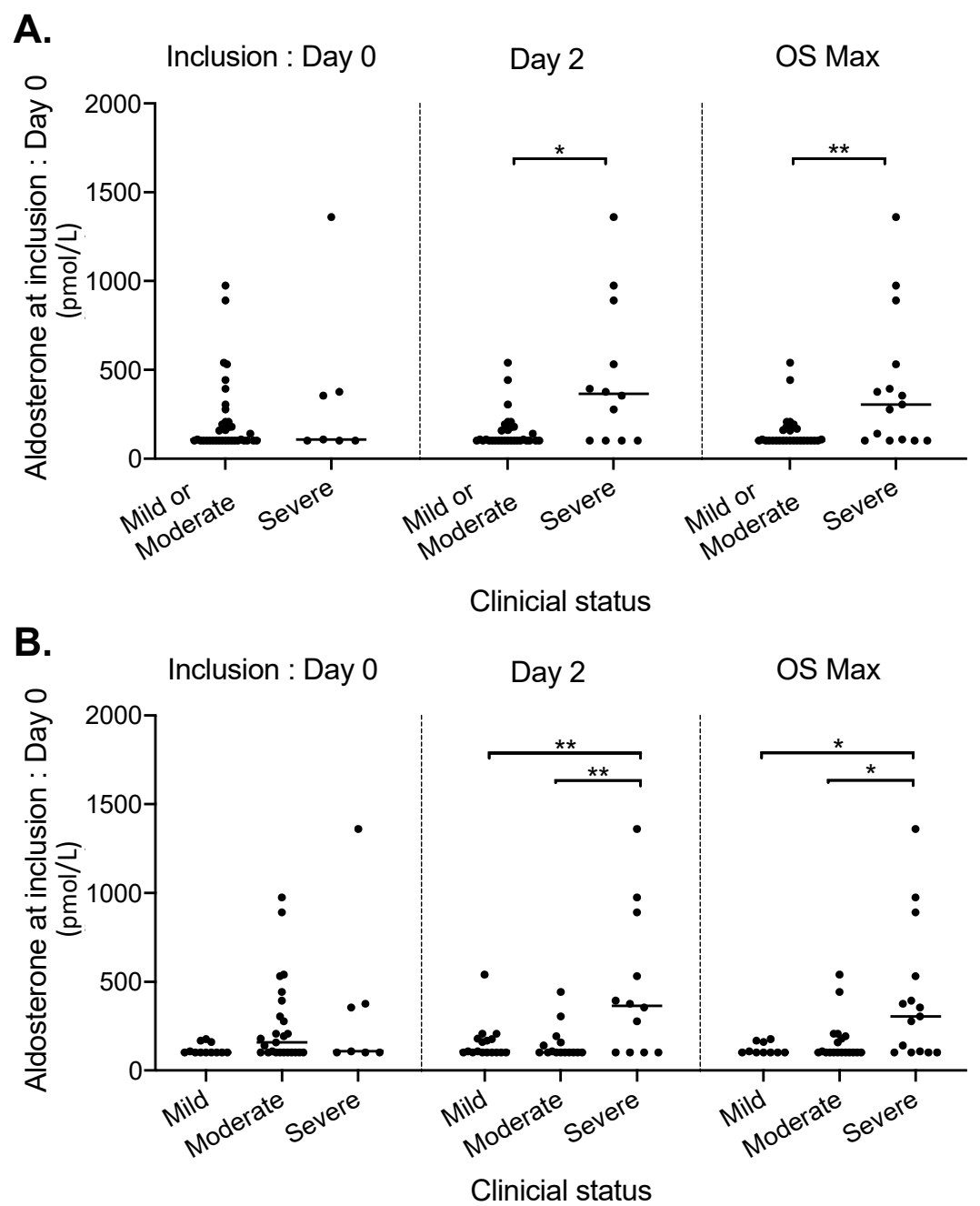

Figure 2. Plasma aldosterone levels at inclusion according to clinical status of patients with Covid-19 infection at Day 0, Day 2 and at the maximum ordinal scale (OS) during the overall period of hospitalization (OS max). The median values are shown (crossbar), in two (A) or three (B) groups related to clinical status severity: mild (OS $\leq 3, n=10)$, moderate (OS $=4, n=10)$ and severe (OS $\geq 5$, $n=15) .{ }^{* *} p<0.01 ;{ }^{*} p<0.05$ (Mann-Whitney test).

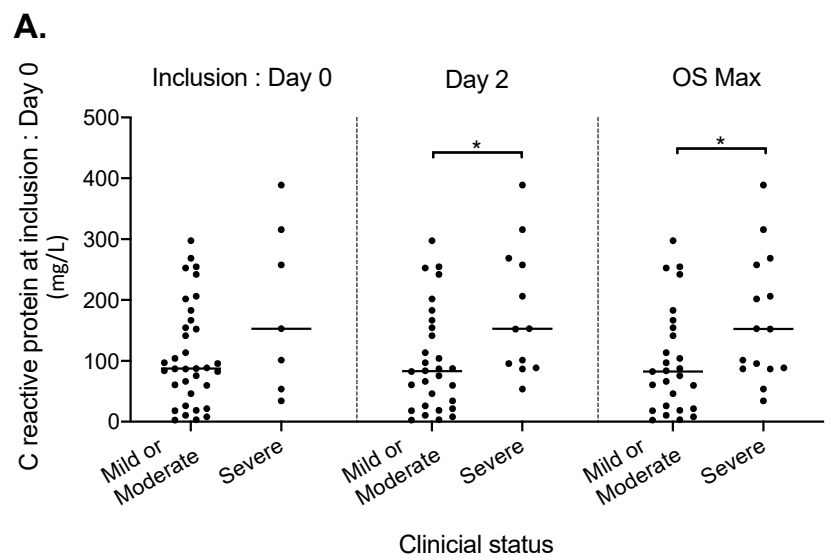

B.

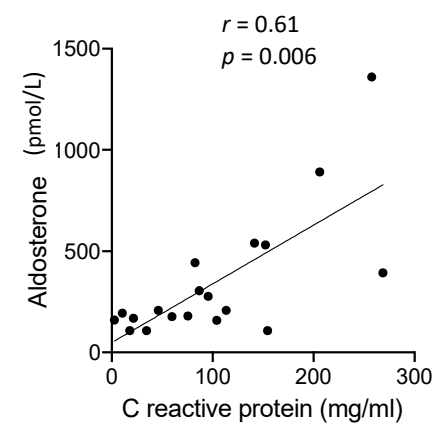

Figure 3. (A) $\mathrm{C}$ reactive protein level at inclusion according to clinical status of patients with Covid-19 infection at Day 0, Day 2, and maximum ordinal scale (OS) during the overall period of hospitalization (OS max). The median values are shown (crossbar), in two groups of clinical status severity: mild or moderate (OS $\leq 4, n=29)$ ) and severe (OS $\geq 5, n=15)$. ${ }^{*} p<0.05$ (Mann-Whitney test). (B) Correlation between aldosterone and $C$ reactive protein at inclusion. 
An additional analysis with CRP level at baseline found concordant results. As compared to patients with mild or moderate clinical status, CRP levels were significantly higher in patients with OS $\geq 5$ on Day 2 or at OS max ( $p=0.01$ and $p=0.02$, respectively) (Figure 3A). Considering the hypothesis that aldosterone may be involved in inflammatory damages of Covid-19, we searched for a relationship between the aldosterone and CRP levels. We conducted this further analysis independently from disease severity (as assessed by the OS), after having excluded the patients who had developed a documented acute bacterial infection in the days close to the biological investigation. Finally, we observed a correlation between aldosterone and CRP levels among patients with an aldosterone level greater than $102.5 \mathrm{pmol} / \mathrm{L}$. In this group of 19 patients, aldosterone level was positively correlated with CRP level at baseline (Spearman coefficient $r$ (95\% CI) $0.61(0.2-0.84), p=0.006$ ) (Figure 3B).

\section{Discussion}

In the present study based upon data collected in the real-life settings of the brutal SARS-CoV-2 outbreak, we report an association between the plasma levels of aldosterone close to admission and the severity of Covid-19 course, as defined by the ordinal scale grade. Indeed, the most severe patients, who required at least intensive care $(\mathrm{OS} \geq 5)$, had significantly higher plasma levels of aldosterone when admitted than those hospitalized in medical units, with $(\mathrm{OS}=4)$ or without $(\mathrm{OS}=3)$ oxygen support. This association appears to be relevant both when considering the OS 2 days after admission and according to the maximal OS during the overall period of hospitalization. In most patients, aldosterone levels remained within a physiological range, but the significant differences observed between groups according to severity were independent of the renin levels and aldosterone/renin ratio. Such a hormonal profile is suggestive of a renin-independent hyperaldosteronism [10], which could be a hallmark of some patients with the most severe forms of Covid-19. Conversely, low aldosterone levels were observed in those with a less severe disease (OS $=3$ or 4$)$. This could be related either to a failure of the aldosterone assay to discriminate within the minimal values or reflect a tendency to adrenal insufficiency. However, this latter hypothesis is not supported by the plasma cortisol levels.

As previously reported [3-5], CRP levels were coherent with the severity of Covid-19, which is characterized by a severe inflammatory syndrome. Interestingly, patients with aldosterone levels higher than $102.5 \mathrm{pmol} / \mathrm{L}$ exhibited a linear relationship between CRP and aldosterone levels. This further finding is in line with the suspected role of the viral load in the ACE/ACE2 imbalance, which occurs before the onset of the cytokine storm [2,11,12]. Indeed, SARS-CoV-2 could disrupt the RAAS through its binding to ACE2, which is the negative regulator of the system [8]. Hence, the defective inactivation of Ang2 could lead to the activation of RAAS, including an increased secretion of aldosterone.

The role of Ang2 in the severity of lung inflammatory damage in Covid-19 is supported by previous investigational reports. First, Imai et al. $[13,14]$ demonstrated in several animal models of acute lung injury (acid inhalation, sepsis or pneumonia) that Ang2 can worsen pulmonary lesions (including inflammatory infiltrates) through the stimulation of the Ang2 type 1 receptor (AT1R). Conversely, ACE2 and Ang2 type 2 receptor (AT2R) can down-regulate these deleterious effects, whereas abrogated ACE2 expression can induce severe respiratory failure in mice models. In addition, the levels of Ang2 are increased in these mice, which exhibit severe lung involvement partially reversible with the pharmacological inhibition of the AT1R [13,14]. During SARS-COV-1 infection, ACE2 knockout mice were resistant to virus infection and their lung samples were devoid of inflammation [15]. In contrast, the binding of the SARS-spike protein to ACE2 downregulates this regulator pathway, leading to severe lung injury and acute respiratory failure, as illustrated in a mouse model by Kuba and Coll [15]. In their study, blocking the RAAS limited the lung injury. These findings are in line with the concept that RAAS disruption could trigger inflammation in Covid-19.

Furthermore, beside coronavirus infections, the potentially deleterious effects of RAAS have been documented in several tissues (including heart and lung) and medical conditions (such as hypertension, heart failure, obesity, etc.) [16] and have been also documented beyond the regulation of sodium, extracellular volume and blood pressure. The mechanisms leading to RAAS toxicity also include 
(i) modulation of the production of pro-inflammatory cytokines (such as TNF alpha and IL-1 by Ang2 [17] and IL-6 by aldosterone [18]), leading potentially to recruitment of mono/macrophages; (ii) induction of fibrosis (through AT1R) [19]; and (iii) induction of vascular toxicity [20] and modulation of angiogenesis [21,22]. In the context of ACE2 neutralization by SARS-CoV-2, all these mechanisms could be exacerbated, while their clinical consequences are more limited in classical conditions of RAAS hyperactivation (such as chronic heart failure, etc. [16]). Importantly, the pathogenic mechanisms of Covid-19 are concordant with autoptic observations and biological findings, which include the cytokine storm (with IL6, IL1, TNF, etc.) [2,11,12], fibrosis [23], endothelitis and modulated angiogenesis [24,25].

In addition, the key role of RAAS toxicity could be also corroborated by the promising beneficial effects observed with anti-aldosterone and RAAS blocker treatments in several experimental conditions of pulmonary diseases [26]. In Covid-19, these protective effects are extensively debated $[8,27,28]$. Finally, the potentially deleterious effects of RAAS may take place in the pathophysiology of Covid-19. From this point of view, our findings suggest that both CRP and aldosterone levels may impact the clinical status. Further studies are required to document and confirm the suspected role of RAAS in Covid-19.

Our study has limitations due to the collection of plasma samples for hormonal assays in an emergency setting related to the admission for Covid-19 acute infection. Hence, optimal standardized conditions for assessing plasma renin and aldosterone levels were not met, and multiple confounding factors could be involved in the modulation of plasma aldosterone secretion. However, when we adjusted for all of these confounding parameters, plasma aldosterone levels remained significantly associated to disease severity.

In the present study, higher plasmatic aldosterone and CRP levels at inclusion are associated with severe clinical course of Covid-19 in hospitalized patients, and both parameters appear to be correlated. Our results suggest that aldosterone levels may reflect the severity of Covid-19, but this remains to be demonstrated at a larger scale. Our findings open new perspectives into the understanding of the contribution of RAAS in Covid-19 and its possible role in the outcomes of Covid-19. Further investigations are awaited to explore more thoroughly the association between increased aldosterone levels, ACE/ACE2 imbalance, inflammatory biomarkers and the severity of the Covid-19 course.

Supplementary Materials: The following are available online at http://www.mdpi.com/2077-0383/9/7/2315/s1, Figure S1: Patients with no RAAS blocker therapy: clinical status course according to aldosterone level at inclusion, $* p<0.05$ (Mann-Whitney test) (A) and laboratory findings of RAAS explorations by severity of clinical status during the overall period of hospitalization (B), Table S1: Clinical characteristics of patients with Covid-19 according to disease severity classified in three groups: mild (OS max $\leq 3)$, moderate (OS max $=4)$ and severe (OS $\max \geq 5$ ).

Author Contributions: O.V.: data collection, design and conceptualization of the study, statistical analysis, drafting and reviewing of the manuscript; D.M.: data collection, investigation, software, design and conceptualization of the study, statistical analysis, supervision, drafting and reviewing of the manuscript; N.M.: methodology, statistical analysis, drafting and reviewing of the manuscript; I.R.: design and conceptualization of the study, drafting and reviewing of the manuscript; N.N.: design and conceptualization of the study, drafting and reviewing of the manuscript; J.-P.C.: data collection, drafting and reviewing of the manuscript; B.J.: data collection, investigation, drafting and reviewing of the manuscript; C.R.: data collection, investigation, drafting and reviewing of the manuscript; V.F.: data collection, investigation, drafting and reviewing of the manuscript; P.F.: data collection, investigation, drafting and reviewing of the manuscript; S.L.: data collection, investigation, drafting and reviewing of the manuscript; P.T.: data collection, investigation, drafting and reviewing of the manuscript; A.K.: data collection, investigation, drafting and reviewing of the manuscript; A.T.J.M.: design and conceptualization of the study, drafting and reviewing of the manuscript; A.M.: data collection, investigation, drafting and reviewing of the manuscript; I.B.: data collection, investigation, drafting and reviewing of the manuscript; R.L.: data collection, investigation, drafting and reviewing of the manuscript; K.K.: data collection, investigation, drafting and reviewing of the manuscript; V.L.M.: data collection, investigation, drafting and reviewing of the manuscript; E.R.: design and conceptualization of the study, methodology, drafting and reviewing of the manuscript; P.G.: design and conceptualization of the study, methodology, statistical analysis, supervision, drafting and reviewing of the manuscript. All authors have read and agreed to the published version of the manuscript.

Funding: This research received no external funding.

Acknowledgments: This work was supported by Montpellier University Hospital, Montpellier University.

Conflicts of Interest: The authors declare no conflict of interest. 


\section{References}

1. Guan, W.-J.; Ni, Z.-Y.; Hu, Y.; Liang, W.-H.; Ou, C.-Q.; He, J.-X.; Liu, L.; Shan, H.; Lei, C.-L.; Hui, D.S.C.; et al. Clinical Characteristics of Coronavirus Disease 2019 in China. N. Engl. J. Med. 2020, 382, 1708-1720. [CrossRef] [PubMed]

2. Bouadma, L.; Lescure, F.-X.; Lucet, J.-C.; Yazdanpanah, Y.; Timsit, J.-F. Severe SARS-CoV-2 infections: practical considerations and management strategy for intensivists. Intensive Care Med. 2020, 46, 579-582. [CrossRef] [PubMed]

3. Zhang, J.; Yu, M.; Tong, S.; Liu, L.-Y.; Tang, L.-V. Predictive factors for disease progression in hospitalized patients with coronavirus disease 2019 in Wuhan, China. J. Clin. Virol. 2020, 127, 104392. [CrossRef] [PubMed]

4. Zhang, X.; Tan, Y.; Ling, Y.; Lu, G.; Liu, F.; Yi, Z.; Jia, X.; Wu, M.; Shi, B.; Xu, S.; et al. Viral and host factors related to the clinical outcome of COVID-19. Nature 2020, 583, 437-440. [CrossRef]

5. Cariou, B.; Hadjadj, S.; Wargny, M.; Pichelin, M.; Al-Salameh, A.; Allix, I.; Amadou, C.; Arnault, G.; Baudoux, F.; Bauduceau, B.; et al. Phenotypic characteristics and prognosis of inpatients with COVID-19 and diabetes: the CORONADO study. Diabetologia 2020, 63, 1500-1515. [CrossRef]

6. Cecconi, M.; Piovani, D.; Brunetta, E.; Aghemo, A.; Greco, M.; Ciccarelli, M.; Angelini, C.; Voza, A.; Omodei, P.; Vespa, E.; et al. Early Predictors of Clinical Deterioration in a Cohort of 239 Patients Hospitalized for Covid-19 Infection in Lombardy, Italy. J. Clin. Med. 2020, 9, 1548. [CrossRef]

7. Li, W.; Moore, M.J.; Vasilieva, N.; Sui, J.; Wong, S.K.; Berne, M.A.; Somasundaran, M.; Sullivan, J.L.; Luzuriaga, K.; Greenough, T.C.; et al. Angiotensin-converting enzyme 2 is a functional receptor for the SARS coronavirus. Nature 2003, 426, 450-454. [CrossRef]

8. Vaduganathan, M.; Vardeny, O.; Michel, T.; McMurray, J.J.V.; Pfeffer, M.A.; Solomon, S.D. Renin-Angiotensin-Aldosterone System Inhibitors in Patients with Covid-19. N. Engl. J. Med. 2020, 382, 1653-1659. [CrossRef]

9. Cao, B.; Wang, Y.; Wen, D.; Liu, W.; Wang, J.; Fan, G.; Ruan, L.; Song, B.; Cai, Y.; Wei, M.; et al. A Trial of Lopinavir-Ritonavir in Adults Hospitalized with Severe Covid-19. N. Engl. J. Med. 2020, 382, 1787-1799. [CrossRef]

10. Sabbadin, C.; Armanini, D. Syndromes that Mimic an Excess of Mineralocorticoids. High Blood Press. Cardiovasc. Prev. 2016, 23, 231-235. [CrossRef]

11. Moore, J.B.; June, C.H. Cytokine release syndrome in severe COVID-19. Science 2020, 368, 473-474. [CrossRef] [PubMed]

12. Vabret, N.; Britton, G.J.; Gruber, C.; Hegde, S.; Kim, J.; Kuksin, M.; Levantovsky, R.; Malle, L.; Moreira, A.; Park, M.D.; et al. Immunology of COVID-19: current state of the science. Immunity 2020, 52, 910-941. [CrossRef] [PubMed]

13. Imai, Y.; Kuba, K.; Rao, S.; Huan, Y.; Guo, F.; Guan, B.; Yang, P.; Sarao, R.; Wada, T.; Leong-Poi, H.; et al. Angiotensin-converting enzyme 2 protects from severe acute lung failure. Nature 2005, 436, 112-116. [CrossRef]

14. Imai, Y.; Kuba, K.; Ohto-Nakanishi, T.; Penninger, J.M. Angiotensin-Converting Enzyme 2 (ACE2) in Disease Pathogenesis. Circ. J. 2010, 74, 405-410. [CrossRef] [PubMed]

15. Kuba, K.; Imai, Y.; Rao, S.; Gao, H.; Guo, F.; Guan, B.; Huan, Y.; Yang, P.; Zhang, Y.; Deng, W.; et al. A crucial role of angiotensin converting enzyme 2 (ACE2) in SARS coronavirus-induced lung injury. Nat. Med. 2005, 11, 875-879. [CrossRef]

16. Patel, S.; Rauf, A.; Khan, H.; Abu-Izneid, T. Renin-angiotensin-aldosterone (RAAS): The ubiquitous system for homeostasis and pathologies. Biomed. Pharmacother. 2017, 94, 317-325. [CrossRef]

17. Watanabe, T.; Miyoshi, M.; Imoto, T. Angiotensin II: its effects on fever and hypothermia in systemic inflammation. Front. Biosci. 2004, 9, 438-447. [CrossRef]

18. Liao, C.-W.; Chou, C.-H.; Wu, X.-M.; Chen, Z.-W.; Chen, Y.-H.; Chang, Y.-Y.; Wu, V.-C.; Rose-John, S.; Hung, C.-S.; Lin, Y.-H.; et al. Interleukin-6 plays a critical role in aldosterone-induced macrophage recruitment and infiltration in the myocardium. Biochim. Biophys. Acta Mol. Basis Dis. 2020, 1866, 165627. [CrossRef] 
19. Chou, C.-H.; Hung, C.-S.; Liao, C.-W.; Wei, L.-H.; Chen, C.-W.; Shun, C.-T.; Wen, W.-F.; Wan, C.-H.; Wu, X.-M.; Chang, Y.-Y.; et al. IL-6 trans-signalling contributes to aldosterone-induced cardiac fibrosis. Cardiovasc. Res. 2018, 114, 690-702. [CrossRef]

20. Pacurari, M.; Kafoury, R.; Tchounwou, P.B.; Ndebele, K. The Renin-Angiotensin-Aldosterone System in Vascular Inflammation and Remodeling. Int. J. Inflamm. 2014, 2014, 1-13. [CrossRef]

21. Wilkinson-Berka, J.L.; Suphapimol, V.; Jerome, J.R.; Deliyanti, D.; Allingham, M.J. Angiotensin II and aldosterone in retinal vasculopathy and inflammation. Exp. Eye Res. 2019, 187, 107766. [CrossRef] [PubMed]

22. Maron, B.A.; Zhang, Y.-Y.; White, K.; Chan, S.Y.; Handy, D.E.; Mahoney, C.E.; Loscalzo, J.; Leopold, J.A. Aldosterone Inactivates the Endothelin-B Receptor via a Cysteinyl Thiol Redox Switch to Decrease Pulmonary Endothelial Nitric Oxide Levels and Modulate Pulmonary Arterial Hypertension. Circulation 2012, 126, 963-974. [CrossRef] [PubMed]

23. Edler, C.; Schröder, A.S.; Aepfelbacher, M.; Fitzek, A.; Heinemann, A.; Heinrich, F.; Klein, A.; Langenwalder, F.; Lütgehetmann, M.; Meißner, K.; et al. Dying with SARS-CoV-2 infection-an autopsy study of the first consecutive 80 cases in Hamburg, Germany. Int. J. Legal Med. 2020, 134, 1275-1284. [CrossRef] [PubMed]

24. Varga, Z.; Flammer, A.J.; Steiger, P.; Haberecker, M.; Andermatt, R.; Zinkernagel, A.S.; Mehra, M.R.; Schuepbach, R.A.; Ruschitzka, F.; Moch, H. Endothelial cell infection and endotheliitis in COVID-19. Lancet 2020, 395, 1417-1418. [CrossRef]

25. Ackermann, M.; Verleden, S.E.; Kuehnel, M.; Haverich, A.; Welte, T.; Laenger, F.; Vanstapel, A.; Werlein, C.; Stark, H.; Tzankov, A.; et al. Pulmonary Vascular Endothelialitis, Thrombosis, and Angiogenesis in Covid-19. N. Engl. J. Med. 2020, 383, 120-128. [CrossRef]

26. Tan, W.S.D.; Liao, W.; Zhou, S.; Mei, D.; Wong, W.-S.F. Targeting the renin-angiotensin system as novel therapeutic strategy for pulmonary diseases. Curr. Opin. Pharmacol. 2018, 40, 9-17. [CrossRef]

27. Aronson, J.K.; Ferner, R.E. Drugs and the renin-angiotensin system in covid-19. BMJ 2020, 369, m1313. [CrossRef]

28. Reynolds, H.R.; Adhikari, S.; Pulgarin, C.; Troxel, A.B.; Iturrate, E.; Johnson, S.B.; Hausvater, A.; Newman, J.D.; Berger, J.S.; Bangalore, S.; et al. Renin-Angiotensin-Aldosterone System Inhibitors and Risk of Covid-19. N. Engl. J. Med. 2020, 382, 2441-2448. [CrossRef]

(C) 2020 by the authors. Licensee MDPI, Basel, Switzerland. This article is an open access article distributed under the terms and conditions of the Creative Commons Attribution (CC BY) license (http://creativecommons.org/licenses/by/4.0/). 